\title{
LMP2A-Specific Cytotoxic T-Lymphocytes
}

National Cancer Institute

\section{Source}

National Cancer Institute. LMP2A-Specific Cytotoxic T-Lymphocytes. NCI Thesaurus.

Code C62784.

A preparation of cytotoxic T-lymphocytes (CTL), specifically reactive to Epstein-Barr virus (EBV) latent membrane protein-2A (LMP2A), with potential antineoplastic activity. T lymphocytes are exposed ex vivo to dendritic cells (DCs) transfected with a replicationdeficient adenovirus encoding EBV LMP2A. Subsequently, LMP2A-specific CT Ls are exposed to EBV infected cells transfected with adenovirus encoding LMP2A, thereby further stimulating CT Ls. Administered to patients with EBV-positive tumors, LMP2Aspecific CT Ls target LMP2A-positive cells, resulting in cell lysis and inhibition of cancer cell proliferation. EBV LMP2A may be expressed in various malignancies, including nasopharyngeal carcinoma and Hodgkin and non-Hodgkin lymphomas. 